\title{
Using Visual Analytics to Determine the Utilization of Preoperative Anesthesia Assessments
}

\author{
J.P. Wanderer'; C.L. Gruss²; J.M. Ehrenfeld ${ }^{3}$ \\ ${ }^{1}$ Departments of Anesthesiology and Biomedical Informatics, Vanderbilt University, Nashville, TN, United States; ${ }^{2}$ Department of \\ Anesthesiology, Vanderbilt University, Nashville, TN, United States; ${ }^{3}$ Departments of Anesthesiology, Biomedical Informatics and \\ Surgery, Vanderbilt University, Nashville, TN, United States
}

\begin{abstract}
Keywords
Visual, analytics, anesthesia

Summary

Background: Preoperative assessments are a required and essential element of anesthetic care, yet little is known about the utilization of these documents by clinicians who are not part of the anesthesia care team. As part of perioperative workflow restructuring, we implemented a data visualization technique of electronic medical record audit log data to understand the utilization of preoperative anesthesia assessments by non-anesthesia personnel.

Methods: An audit log cache containing 140 days of data was queried for all accesses of preoperative anesthesia assessment documents for any patient who had a preoperative anesthesia assessment that was accessed during that period. User roles were aggregated into categories. Descriptive statistics and data visualization were generated using R (R Software Foundation, Vienna, Austria). Comparisons were performed with the Wilcoxon signed rank test with continuity correction.

Results: During the study period, 73802 (0.015\%) of the 485062902 audit log accesses were preoperative anesthesia assessments representing 412 departments, 302 user roles, and 3916 distinct users who accessed preoperative anesthesia assessments from 14235 surgical cases. Each assessment was accessed 2.9 times on average. Assessments performed in the preoperative anesthesia assessment clinic were accessed more frequently than those created on the day of surgery in the preoperative holding room $(3.58 \pm 5.18$ v. $1.98 \pm 1.76$ average views; $p<0.0001)$. We observed accesses of these documents by pathology and general surgery researchers, as well as orthopedics attending physicians accessing documents that were two years old.

Conclusions: This approach revealed patterns of utilization that had not been previously identified, including usage by surgical residents, surgical faculty, and pathology researchers both before and after the surgical event for which the documents are generated. Knowledge of these dependencies directly informed perioperative workflow restructuring efforts. This visual analytic approach could be broadly utilized to understand documentation dependencies in a variety of clinical contexts.
\end{abstract}

\section{Correspondence to:}

Jonathan P. Wanderer, MD, MPhil

1301 Medical Center Drive, Suite 4648

The Vanderbilt Clinic, Nashville, TN 37232

Tel.: +1 (615) 936-5194

Fax: +1 (615) 936-6493

Email: Jonathan.P.Wanderer@vanderbilt.edu

\author{
Appl Clin Inform 2015; 6: 629-637 \\ http://dx.doi.org/10.4338/ACI-2015-02-CR-0022 \\ received: March 10, 2015 \\ accepted in revised form: August 19, 2015 \\ published: October 21, 2015 \\ Wanderer JP, Gruss CL, Ehrenfeld JM. Using visual ana- \\ lytics to determine the utilization of preoperative anes- \\ thesia assessments. Appl Clin Inform 2015; 6: 629-637 \\ http://dx.doi.org/10.4338/ACl-2015-02-CR-0022
}




\section{Introduction}

Preoperative assessment of surgical patients by a clinic-based anesthesia team in advance of elective surgery has been demonstrated to reduce case cancellations on the day of surgery, improve the efficiency of clinical operations and lead to patient satisfaction [1-3]. These benefits are largely seen with complex patients or those undergoing more invasive surgeries, as healthier patients undergoing minor surgery can typically be evaluated on the morning of surgery without an increased risk of cancellations or delays [4]. This assessment is sometimes the only comprehensive history and physical that a surgical patient receives and can be a reliable source of patient information. As part of a perioperative workflow restructuring initiative at our institution, we evaluated closing our preoperative clinic which performs preoperative history and physical exams, and replacing it with a system where preoperative history and physical exams would not be completed until immediately prior to surgery. Thus comprehensive patient information would not be available prior to the day of surgery, with unknown consequences on workflow. Prior to making this shift, we sought to assess the current utilization of this information.

As part of the Health Information, Privacy and Portability Act, health care institutions are required to maintain audit log data that record all accesses of electronic medical records [5]. In addition to meeting this requirement, these data have been used for other purposes as well, including understanding the life cycle of electronic medical record documents [6], exploring trainee utilization of the electronic medical record [7], and for medicolegal purposes [7, 8], in addition to their primary purpose of addressing patient privacy concerns. As a consequence, these logs represent a detailed, rich source of information that could describe how electronic medical record documents are actually utilized within an institution, and is a data source that is required by law to be maintained. Thus, these logs are well-suited to inform workflow analysis.

Visual analytics in healthcare have been used to deal with the information overload that is encountered when dealing with large volumes of data generated by electronic medical records [9]. Newer techniques have been developed to look at individual patients, as well as larger populations, over time $[10,11]$. In recent history, visual analytics has even been utilized to optimize drug-drug interaction alert rules [12] and model patient severity in intensive care units [13]. Audit logs are no exception to this information overload challenge, as multiple audit log records can be generated by every interaction that personnel have with an electronic medical record. Techniques have been described to filter through this data source to identify accesses that may be concerning from a privacy perspective $[14,15]$; however, these algorithms are not suited to inform workflow analysis.

\section{Objectives}

Few data exist describing the utilization of routinely generated electronic clinical documentation even though $67 \%$ of US academic hospitals reported use of automated electronic anesthesia records in May 2013 (16). These electronic records have the potential to serve as a huge reservoir of information for perioperative patient care. In this article, our goal is to understand the utilization of preoperative anesthesia assessments by non-anesthesia personnel, to leverage audit logs to inform workflow restructuring, and to demonstrate how visual analytics can be used to efficiently identify patterns within large data sets.

\section{Methods}

The Institution Review Board of the Vanderbilt University Medical Center approved this study. Additionally, approval was sought and obtained for this analysis from the home institution's privacy office. The audit logs for our electronic medical record were queried from the audit log cache in our data warehouse, which contains 140 days of data. We queried that source for all accesses of our preoperative anesthesia assessment documents between January 24, 2013 and June 13, 2013. While the audit log cache contains 140 days of data, each preoperative assessment is retained permanently as part of that patient's EMR (Electronic Medical Record). Therefore, during the 140-day cache period 
it is possible to see accesses of documents of any age. We included all patients who had a preoperative anesthesia assessment that was accessed between these dates.

For each user associated with an audit log access, we determined the user's role and department assignment. Due to privacy concerns, we only analyzed these accesses in aggregate by user role and department without the identification of individual users. We identified users who worked in the preoperative holding room area, post-anesthesia care unit, operating room, preoperative anesthesia assessment clinic and anesthesia department, and excluded their accesses from analysis. These users, again, were identified by their role and department assignment.

Using a unique identifier associated with these documents, we determined the day of surgery and calculated the interval in days between the electronic medical record access of the preoperative assessment and the day of surgery, which we termed access day relative to surgery. Finally, we determined if the preoperative assessment occurred in a preoperative clinic visit or on the day of surgery in the preoperative holding room.

To develop a data visualization plot of the audit log accesses, we determined the top 30 departments by total number of accesses and created user role groups. User roles were aggregated into seven categories: nurse practitioner, faculty, coding, case manager, research, resident, or registered nurse. Individual accesses were visualized for the top departments by displaying the access day relative to surgery, grouped by department and color-coded by user role group. Two plots were generated: one that displayed all points to demonstrate all usage patterns, and a second with a point transparency setting of $90 \%$ to provide a visualization that accounted for overplotting. A color palette was selected that allows for full interpretation for those with red-green color vision deficiency. Descriptive statistics and data visualization were generated using R, using the ggplot2 package (R Software Foundation, Vienna, Austria). Normality was assessed using the KolmogorovSmirnov test, and non-parametric comparisons were performed with the Wilcoxon signed rank test with continuity correction. An a level of 0.05 was taken as reference to detect statistical significance in all analyses.

\section{Results}

During the study period, 485062902 audit log accesses were identified over 140 days. Of these, $73802(0.015 \%)$ were accesses of preoperative anesthesia assessments. After removing accesses associated with anesthesia personnel, in the 41036 accessed that remained, we identified 412 departments and 302 user roles. We identified 3916 distinct users who accessed preoperative anesthesia assessments from 14235 surgical cases. Each assessment was accessed 2.9 times on average. Assessments performed in the preoperative anesthesia assessment clinic were accessed more frequently than those created on the day of surgery in the preoperative holding room (28355 vs 12681 total views; $3.58 \pm 5.18$ vs $1.98 \pm 1.76$ average views; $\mathrm{p}<0.0001)$.

We analyzed the user roles that accessed the preoperative anesthesia assessments the most and determined the most frequent access day relative to surgery ( $>$ Table 1 ). This information was supplemented by our data visualization ( $>$ Figure 1 ), which together revealed several patterns of utilization described below.

The user role associated with the most accesses was coding personnel, who accessed these documents most frequently 5 days after surgery ( Table 1 ). This was a known usage of preoperative anesthesia assessments, as these documents are checked prior to anesthesia billing and to supplement comorbidity coding for hospital billing. Surgical residents and surgical nurse practitioners frequently accessed these documents on the day of surgery from departments including general surgery, which was also expected. However, we observed accesses of these documents from pathology researchers, which occurred most frequently the day prior to surgery (black oval, $>$ Figure 1). Similar access patterns were found from surgical nurse practitioners, residents, and attending physicians from other surgical departments. We saw a large cluster of general surgery researcher accesses of documents created 5 years previously (red oval, Figure 1), and a similar cluster from orthopedics and rehabilitation attending physicians accessing documents that were two years old (blue oval, $>$ Figure 1). In order to highlight the most frequent access patterns, we additionally visualized these data with partial transparency to account for overplotting ( $>$ Figure 2). 


\section{Discussion}

In this study, we demonstrated the use of visual analytics to examine a large data set of audit log access records. We found that despite these documents being authored by anesthesia teams, they are frequently accessed by non-anesthesia personnel representing a diverse set of departments and user roles. In conjunction with descriptive statistics, this approach identified several previously unrecognized uses for preoperative anesthesia assessments. Using only descriptive statistics, key pieces of information would have been overlooked such as pattern of use over extended periods of time. Using visual analytics, the use of anesthesia preoperative assessments 2-5 years after their creation became readily apparent. Additionally, the use of this visual approach made it easier to identify that the personnel viewing the records changes in an interesting way over time. Analyses such as this one demonstrate the value of audit logs in understanding workflow, and the specific role that visual analytics can have in identifying patterns within large data sets.

Prior to the results of this analysis, our institution had planned to restructure perioperative workflow to shift patients from preoperative clinic assessment to assessment on the day of surgery. During planning meetings with surgical department heads, it was stated that documents authored by anesthesia teams were not used by members of the surgical departments as part of their workflow. Quantitative analysis revealed that this was not the case, but rather these documents were frequently accessed by a wide variety of non-anesthesia personnel. Most likely, these documents were accessed to provide a comprehensive understanding of patients' medical history, although this is speculation. Following the completion of this study, the departments involved decided to abandon the proposal to close the preoperative clinic. Instead, the preoperative evaluation model was shifted. While the majority of visits are performed as in-person assessments, a portion of the history and physical assessments were converted to histories only, and a small fraction of patients - those without significant past medical history - are evaluated through day of surgery assessments. The type of assessment that each patient receives depends on patient comorbidities and type of procedure, and is specified through VPEC (Vanderbilt Preoperative Evaluation Center) protocols that were developed. Additionally, given the demonstrated need for preoperative information, the timing of assessments relative to surgery was altered. Assessments are now performed well in advance of surgery to allow adequate time for any interested party to review them prior to the day of surgery.

Previously published work related to audit log accesses has largely related to identifying patterns suspicious for privacy violations $[14,15,17]$. Although audit logs have been used to understand care teams related to obstetric and neonatal care [18], to these authors' knowledge they have not previously been utilized to inform workflow restructuring.

This study has several limitations. While we are able to track access of electronic medical record documents, there is no mechanism to determine the value that viewing these documents gave to individual users. It is possible that these documents were accessed but not read. Additionally, because we analyzed these data in aggregate only due to privacy concerns, we were not able to contact users to determine why they were viewing these documents, nor were we able to perform detailed analyses on the clusters identified. Finally, it is a common practice at our institution for clerical staff to print anesthesia preoperative assessments the evening prior to surgery when available to include them as a part of the patient's surgical paper chart. As it is not possible to track usage of these paper copies, our analysis may underestimate the utilization of these documents. However, these paper copies are typically used by anesthesia personnel and perioperative nurses that were excluded from this analysis, minimizing the impact of any underestimation.

\section{Conclusion}

This study enabled a better understanding of the utilization of preoperative anesthesia assessments by non-anesthesia personnel, provided audit log information to impact workflow restructuring, and demonstrated how visual analytics can be used to efficiently identify patterns within large data sets. As maintaining audit logs is required by law, this approach represents one which could be broadly utilized in a variety of clinical settings and for various administrative and clinical purposes. 


\section{Clinical Relevance Statement}

We present an application of a data visualization technique that uses electronic medical record audit $\log$ data to understand the utilization of preoperative anesthesia assessments by nonanesthesia personnel. We demonstrate the utility of an audit log review in potential workflow restructuring and how visual analytics can be employed to identify patterns for large data sets in clinical practice.

\section{Conflict of Interest}

The authors declare that they have no conflicts of interest in the research.

\section{Human Subjects Protection}

The study was performed in compliance with the World Medical Association Declaration of Helsinki on Ethical Principles for Medical Research Involving Human Subjects, and was reviewed by Vanderbilt University Medical Center Institutional Review Board.

\section{Funding}

Dr. Wanderer is supported by the Foundation for Anesthesia Education and Research (FAER) and the Anesthesia Quality Institute (AQI), Mentored Research Training Grant in Health Services Research (MRTG-HSR).

\section{Acknowledgements}

The authors would like to thank the Enterprise Data Warehouse team, the Vanderbilt Anesthesiology and Perioperative Informatics Research Division and Terri Hartman, Director of the Privacy Office at Vanderbilt University Medical Center. 


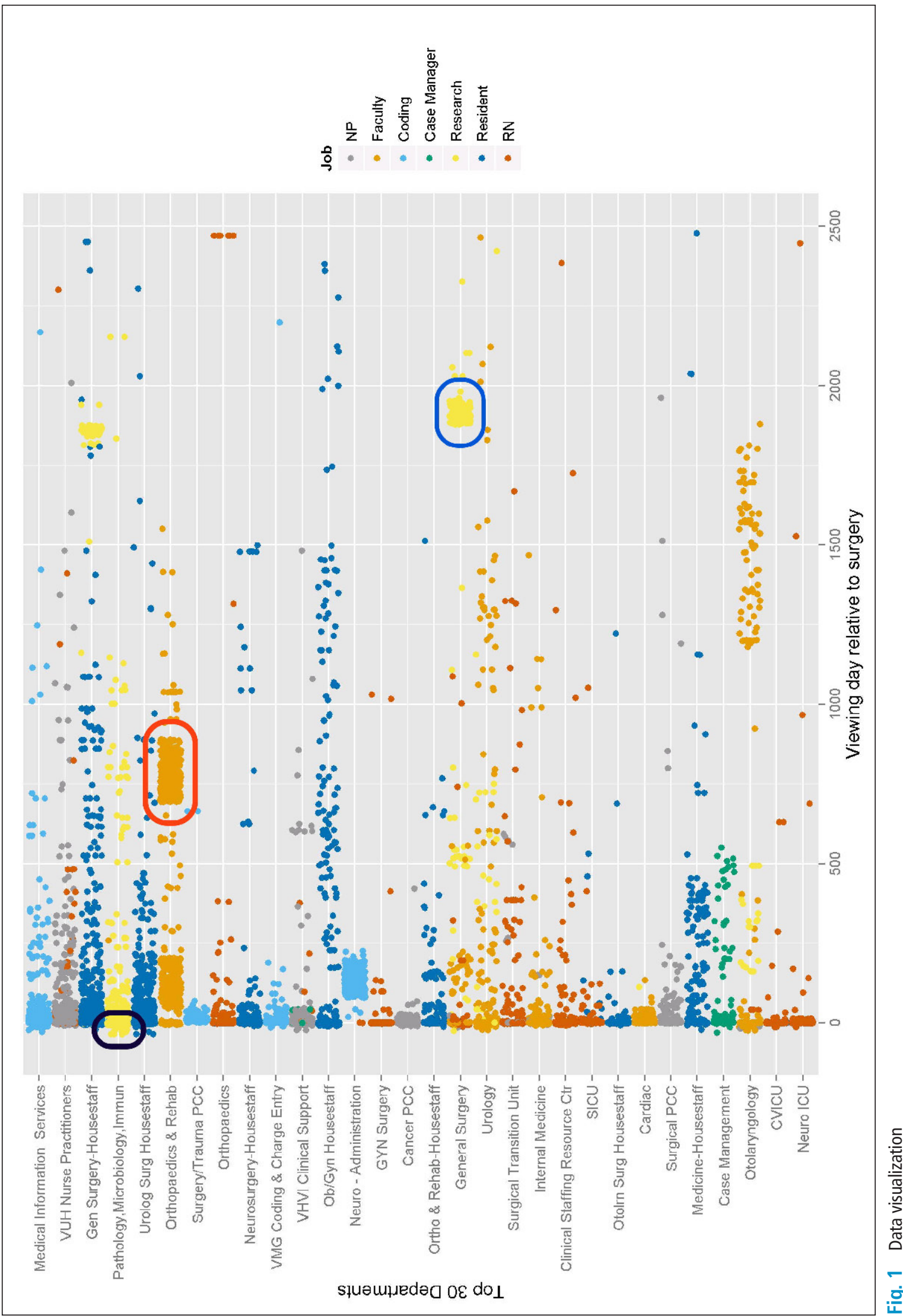

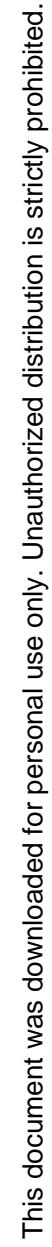




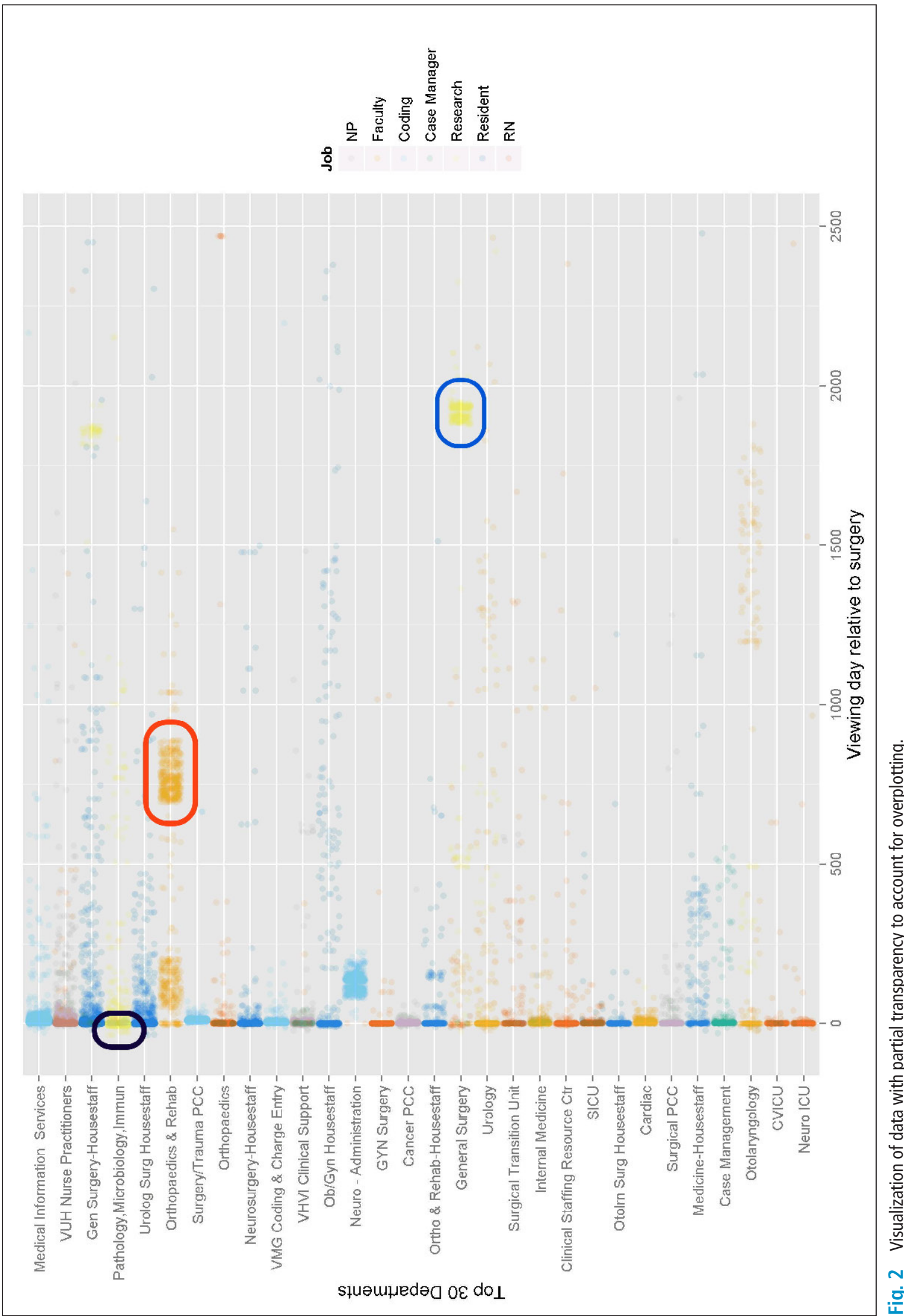


Table 1 Most frequent utilizers of preoperative anesthesia assessments, by distinct user role and department, listed with total count of accesses and most frequent difference between access day and day of surgery.

\begin{tabular}{|l|l|l|l|}
\hline User role & Department & $\begin{array}{l}\text { Access } \\
\text { count }\end{array}$ & $\begin{array}{l}\text { Most frequent difference } \\
\text { between access day and day } \\
\text { of surgery }\end{array}$ \\
\hline Coding & Medical Information Systems & 4526 & 5 \\
\hline Nurse practitioner & VUH Nurse Practitioners & 2960 & 0 \\
\hline Resident physician & General Surgery Housestaff & 1889 & 0 \\
\hline $\begin{array}{l}\text { Clinical documentation } \\
\text { coordinator }\end{array}$ & Medical Information Services & 1784 & 1 \\
\hline Research & Pathology, Microbiology and & 1597 & -1 \\
\hline Resident physician & Immunobiology & 1559 & 0 \\
\hline Health data coordinator & Medical Information Systems & 920 & 1 \\
\hline Quality consultant & Center for Clinical Improvement & 770 & 116 \\
\hline Medical records analyst & Medical Information Systems & 731 & 5 \\
\hline Attending physician & Orthopedics and Rehabilitation & 713 & 737 \\
\hline Coding & Surgery/Trauma & 662 & 10 \\
\hline Resident physician & Neurosurgery Housestaff & 552 & 0 \\
\hline Registered nurse & Orthopedics & 552 & 0 \\
\hline Tumor registrar & Cancer Registry & 536 & 252 \\
\hline Coding & VMG Coding & 498 & 2 \\
\hline Nurse practitioner & VHVI Clinical Support & 408 & -1 \\
\hline Resident physician & Ob/Gyn Housestaff & 402 & 0 \\
\hline $\begin{array}{l}\text { Performance improvement } \\
\text { analyst }\end{array}$ & Center for Clinical Improvement & 400 & 110 \\
\hline Coding & Neurosurgery & 378 & 7 \\
\hline Registered nurse & Gyn Surgery & 0 \\
\hline & & 374 & \\
\hline
\end{tabular}




\section{References}

1. Ferschl MB, Tung A, Sweitzer B, Huo D, Glick DB. Preoperative clinic visits reduce operating room cancellations and delays. Anesthesiology 2005; 103(4): 855-859.

2. Cantlay KL, Baker S, Parry A, Danjoux G. The impact of a consultant anaesthetist led pre-operative assessment clinic on patients undergoing major vascular surgery. Anaesthesia 2006; 61(3): 234-239.

3. Hepner DL, Bader AM, Hurwitz S, Gustafson M, Tsen LC. Patient satisfaction with preoperative assessment in a preoperative assessment testing clinic. Anesth Analg 2004; 98(4): 1099-1105.

4. Sweitzer BJ. Preoperative screening, evaluation, and optimization of the patient's medical status before outpatient surgery. Current opinion in anaesthesiology 2008; 21(6): 711-718.

5. Walsh T, Ahima. Security audits of electronic health information (updated). Journal of AHIMA / American Health Information Management Association 2011; 82(3): 46-50.

6. Hripcsak G, Vawdrey DK, Fred MR, Bostwick SB. Use of electronic clinical documentation: time spent and team interactions. J Am Med Inform Assoc 2011; 18(2): 112-117.

7. McLean TR, Burton L, Haller CC, McLean PB. Electronic medical record metadata: uses and liability. J Am Coll Surg 2008; 206(3): 405-411.

8. Vigoda MM, Lubarsky DA. Failure to recognize loss of incoming data in an anesthesia record-keeping system may have increased medical liability. Anesth Analg 2006; 102(6): 1798-1802.

9. Simpao AF, Ahumada LM, Galvez JA, Rehman MA. A review of analytics and clinical informatics in health care. J Med Syst 2014; 38(4): 45.

10. Shahar Y, Cheng C. Intelligent visualization and exploration of time-oriented clinical data. Topics in health information management 1999; 20(2): 15-31.

11. Klimov D, Shahar Y. A framework for intelligent visualization of multiple time-oriented medical records. AMIA Annu Symp Proc 2005; 2005: 405-409.

12.Simpao AF, Ahumada LM, Desai BR, Bonafide CP, Galvez JA, Rehman MA, Jawad AF, Palma KL, Shelov ED. Optimization of drug-drug interaction alert rules in a pediatric hospital's electronic health record system using a visual analytics dashboard. J Am Med Inform Assoc 2015; 22(2): 361-369.

13. Joshi R, Szolovits P. Prognostic physiology: modeling patient severity in Intensive Care Units using radial domain folding. AMIA Annu Symp Proc. 2012; 2012: 1276-1283.

14. Boxwala AA, Kim J, Grillo JM, Ohno-Machado L. Using statistical and machine learning to help institutions detect suspicious access to electronic health records. J Am Med Inform Assoc 2011; 18(4): 498-505.

15. Kim J, Grillo JM, Boxwala AA, Jiang X, Mandelbaum RB, Patel BA, Mikels D, Vinterbo SA, Ohno-Machado L. Anomaly and signature filtering improve classifier performance for detection of suspicious access to EHRs. AMIA Annu Symp Proc. 2011; 2011: 723-731.

16.Stol IS, Ehrenfeld JM, Epstein RH. Technology diffusion of anesthesia information management systems into academic anesthesia departments in the United States. Anesthesia and analgesia 2014; 118(3): 644-650.

17. Malin B, Nyemba S, Paulett J. Learning relational policies from electronic health record access logs. J Biomed Inform 2011; 44(2): 333-342.

18. Gray JE, Feldman H, Reti S, Markson L, Lu X, Davis RB, Safran CA. Using Digital Crumbs from an Electronic Health Record to identify, study and improve health care teams. AMIA Annu Symp Proc 2011; 2011: 491-500. 